Die FDP- und die Grünen-Wähler sind bei den 25- bis 34-Jährigen, wo sie überproportional gut abgeschnitten haben, am stärksten. Das Elektorat der SPD hat sich leicht verjüngt. Nirgendwo fallen die Abweichungen so eklatant wie bei der NPD aus: Diese ist eine „Männerpartei“ und eine Partei der "Jungen“.

(8) Der formale Bildungsgrad der CDU-Wähler ist unterdurchschnittlich, jener der Wähler der Grünen und der Liberalen überdurchschnittlich. Die CDU ist bei Rentnern überproportional vertreten, die Linke bei Arbeitslosen, die SPD bei Studenten, die FDP bei Selbständigen, Bündnis 90/Grüne bei Angestellten und Studenten, die NPD bei Arbeitslosen und Arbeitern. Wie bei früheren Wahlen geht Gewerkschaftszugehörigkeit mit einem höheren Anteil an Wählern aus den Reihen der Linken und der SPD einher, Konfessionszugehörigkeit mit CDU-Loyalität.

(9) Im Gegensatz zum Ausgang der Landtagswahlen in Thüringen und im Saarland am selben Tag konnte das „,bürgerliche“ Lager das Ergebnis als ein vorgezogenes Votum für den Bund bezeichnen. In Sachsen liegt es bei allen Wahlen seit 1990 mit über 30 Punkten klar vor dem rot-grünen Lager. Trotz dieser eklatanten Asymmetrie kam 2004 eine schwarz-gelbe Koalition nicht zustande, weil PDS und NPD 32,2 Prozent der Stimmen auf sich zu vereinigen vermochten - und vorher nicht wegen der absoluten Mehrheit für die Sächsische Union. Sachsen ist damit - wie kein anderes ostdeutsches Bundesland ${ }^{50}$ - durch eine „bürgerliche“ Dominanz gekennzeichnet.

(10) Die Regierungsbildung verlief komplikationslos und in Windeseile, da CDU und FDP ein Signal für die Bundestagswahl geben wollten. Dass jetzt kein Minister aus dem Kabinett Milbradt von 2004 mehr im Amt ist, zeigt das Machtbewusstsein Tillichs. Bei allen vier Oppositionsparteien wechselten aus unterschiedlichen Gründen die Landesvorsitzenden. Eine Annäherung der SPD und der Grünen an die Linke mit Blick auf die nächste Wahl ist möglich, aber nicht wahrscheinlich, wohl auch von der bundespolitischen Konstellation abhängig.

50 Vgl. Eckhard Jesse, Bundestags-, Landtags- und Europawahlen in den neuen Bundesländern seit 1990, in: Deutschland Archiv, 42. Jg. (2009), H. 6, S. $965-972$.

\title{
Die saarländische Landtagswahl vom 30. August 2009: Auf dem Weg nach Jamaika
}

Jürgen R. Winkler

\section{Die Ausgangslage}

Von 1955 bis 1975 hatte die CDU im kleinsten Flächenstaat der Bundesrepublik ihren Stimmenanteil bei den saarländischen Landtagswahlen kontinuierlich bis auf 49,2 Prozent steigern können. Ebenso wie auf Bundesebene hatte sich in den ersten zwei Jahrzehnten nach der Eingliederung des Landes in die Bundesrepublik auch im Saarland ein hoch kon- 
zentriertes symmetrisches Parteiensystem mit zwei Großparteien herausgebildet ${ }^{1}$. Dem Aufstieg folgte jedoch bald ein drastischer Niedergang. Bei drei aufeinander folgenden Landtagswahlen musste die CDU derart große Verluste verkraften, dass sie im Jahr der Vereinigung schließlich 21 Punkte hinter der SPD zurücklag. Die schwere Krise in der Stahlindustrie und die hohe Arbeitslosigkeit beendeten vorläufig die Vormacht der CDU an der Saar. Nachdem die SPD 1985 erstmals die absolute Mehrheit der Mandate erobert hatte, nahm sie in den folgenden 14 Jahren eine mit großer Macht ausgestattete Stellung ein. Unter der Führung Oskar Lafontaines gewann sie dreimal in Folge die absolute Mehrheit der Mandate im Landtag, womit sie zeitweise zur dominanten Partei im saarländischen Parteiensystem aufsteigen konnte. Parallel zum Niedergang der CDU hatte sich erstmals seit der Eingliederung in die Bundesrepublik ein asymmetrisches Parteiensystem herausgebildet.

Mitte der 1990er Jahre gelang es Klaus Töpfer, den Niedergang der saarländischen CDU zu stoppen und den Grundstein für den Wiederaufstieg zu legen, der sich schließlich unter der Führung von Peter Müller vollzog. Der Unmut über Bundeskanzler Gerhard Schröders Wirtschafts- und Sozialpolitik schuf ein positives Meinungsklima für die CDU und stärkte Peter Müller den Rücken. Im Schatten der Bundespolitik konnte er sein Profil in der Wahrnehmung der Saarländer schärfen und Vertrauen auch bei Wählern gewinnen, die ihm zuvor skeptisch gegenübergestanden hatten. So war eine große Mehrheit der Bürger lange mit seiner Regierungsarbeit zufrieden ${ }^{2}$. Erst während der Großen Koalition im Bund trübte sich die Stimmung an der Saar wieder deutlich ein. Das Bild von der CDU und die Beurteilung ihrer Regierungsleistung verschlechterten sich deutlich. Auch das positive Image des Ministerpräsidenten begann zu bröckeln. Waren 2004 noch drei Viertel der Bürger mit seiner Arbeit zufrieden, war die Zustimmung im Vorfeld der Landtagswahl 2009 auf etwas

1 Vgl. zur Struktur und Entwicklung des Parteiensystems im Saarland Jürgen R. Winkler, Das Parteiensystem des Saarlandes, in: Uwe Jun / Melanie Haas / Oskar Niedermayer (Hrsg.), Parteien und Parteiensysteme in den deutschen Ländern, Wiesbaden 2008, S. 369 - 386.

2 Vgl. zu den früheren Landtagswahlen im Saarland: Jürgen W. Falter, Faktoren der Wahlentscheidung. Eine wahlsoziologische Analyse am Beispiel der saarländischen Landtagswahl 1970, Köln u.a. 1973; Adolf Kimmel, Die saarländische Landtagswahl vom 4. Mai 1975. Erosion im sozialliberalen Bündnis?, in: ZParl, 6. Jg. (1975), H. 4, S. 498 - 508; ders., Die saarländische Landtagswahl vom 27. April 1980. Gefährdung der „bürgerlichen” Koalition?, in: ZParl, 11. Jg. (1980), H. 2, S. 222 - 237; ders., Die saarländische Landtagswahl vom 10. März 1985: Zwei Verlierer, zwei Gewinner, ein Sieger oder: Der Wähler hat den Wechsel gewollt, in: ZParl, 16. Jg. (1985), H. 3, S. 322 - 337; Eberhard Sandschneider, Die saarländische Landtagswahl vom 28. Januar 1990: Ein Sieg der SPD ohne bundespolitische Signalwirkung, in: ZParl, 21. Jg. (1990), H. 3, S. 418 - 429; Jürgen R. Winkler, die saarländische Landtagswahl vom 16. Oktober 1994: Bestätigung der SPD-Mehrheit, Debakel der FDP, in: ZParl, 26. Jg. (1995), H. 2, S. 249 - 261; ders., Die saarländische Landtagswahl vom 5. September 1999. Die CDU erhält die Macht zurück, in: ZParl, 31. Jg. (2000), H. 1, S. 28 - 42; ders., Die saarländische Landtagswahl vom 5. September 2004. Vom Zwei- zum Vierparteiensystem mit einer dominanten CDU, in: ZParl, 36. Jg. (2005), H. 1, S. 19 - 35; Forschungsgruppe Wahlen e.V., Wahl im Saarland. Eine Analyse der Landtagswahl vom 27. April 1980, Mannheim 1980; dies., Wahl im Saarland. Eine Analyse der Landtagswahl vom 10. März 1985, Mannheim 1985; dies., Wahl im Saarland. Eine Analyse der Landtagswahl vom 28. Januar 1990, Mannheim 1990; dies., Wahl im Saarland. Eine Analyse der Landtagswahl vom 16. Oktober 1994, Mannheim 1994; dies., Wahl im Saarland. Eine Analyse der Landtagswahl vom 5. September 1999, Mannheim 1999; dies., Wahl im Saarland. Eine Analyse zur Landtagswahl vom 5. September 2004, Mannheim 2004. 
mehr als die Hälfte gefallen. Der Wandel des Meinungsklimas führte schließlich dazu, dass die CDU Saar keine Alleinregierung mehr, sondern eine „bürgerliche Mehrheit“ bei der Landtagswahl anzustreben begann.

Nachdem Oskar Lafontaine seine Ämter als Bundesfinanzminister und als Bundesvorsitzender der SPD hingeworfen und sich ins Privatleben zurückgezogen hatte und sein Nachfolger im Vorsitz der saarländischen SPD Reinhard Klimmt affärenbedingt zurückgetreten war, konnte Heiko Maas zur neuen Führungspersönlichkeit aufsteigen. Mit ihm war die SPD bei den Landtagswahlen 2004 zwar dramatisch abgestürzt, doch glaubte die Partei nach ihrem Wahlerfolg in Hessen Anfang 2008 erneut an die Chance, die CDU von der Macht verdrängen zu können. Auch die in den Umfragen gemessenen Werte hellten sich für die Sozialdemokraten insoweit wieder auf, als sich die Kluft zur CDU verringerte und ihr Spitzenkandidat in der Wahrnehmung der Bürger aufholte. Am Ende konnten sich ebenso viele Saarländer Heiko Maas als Ministerpräsidenten vorstellen wie Peter Müller. Trotz dieser positiven Entwicklung befand sich die SPD bei der Landtagswahl im Herbst 2009 in einer noch ungünstigeren Ausgangslage als bei der vorangegangenen Wahl. Mit der Kandidatur der Linkspartei unter der Führung des früheren SPD-Vorsitzenden und saarländischen Ministerpräsidenten Oskar Lafontaine trat ihr nämlich wie schon bei der Bundestagswahl 2005 eine ernsthafte Konkurrentin im linken Wählersegment gegenüber.

Die FDP war in den 1990er Jahren drastisch eingebrochen, bei zwei Landtagswahlen an der Fünfprozenthürde gescheitert und erst 2004 mit 5,2 Prozent der Stimmen wieder knapp in den Saarländischen Landtag eingezogen. Obwohl die Freidemokraten ihre strukturellen Probleme nicht beheben konnten, verbesserte sich ihre Ausgangslage mit der Bildung der Großen Koalition im Bund zunehmend ${ }^{3}$. Sie schickten erneut Christoph Hartmann als Spitzenkandidaten ins Rennen. Da die FDP in der öffentlichen Meinung seit der Bildung der Großen Koalition wieder deutlich positiver wahrgenommen wurde, war ihre Ausgangslage an der Saar diesmal so günstig wie lange nicht mehr. Das primäre Ziel der FDP bestand darin, mit Peter Müller zusammen eine „bürgerliche“ Regierung zu bilden.

Nach drei erfolglosen Versuchen hatten die Grünen 1994 erstmals den Einzug in den Landtag geschafft, waren aber 1999 erneut an der Fünfprozenthürde gescheitert, da viele ihrer Wähler wieder zur SPD gewandert waren. Bei der Landtagswahl 2004 gelang ihnen erst zum zweiten Mal knapp der Einzug in den Landtag, wo sie jedoch keine nennenswerten Akzente zu setzen vermochten. Als ihren Spitzenkandidaten schickten sie 2009 Hubert Ulrich ins Rennen, der auch schon fünf Jahre zuvor diese Funktion bekleidet hatte. Angesichts der Wahlgeschichte bestand das primäre Ziel der Grünen darin, erneut in das Parlament einzuziehen. Obwohl sie keine Koalitionsaussage gemacht hatten, wünschten sie sich eine Regierungsbeteiligung. Und weil eine Mehrheit von SPD und Grünen auch aus ihrer Sicht völlig unrealistisch war, favorisierten sie nach außen eine Ampel mit der SPD und den Freien Demokraten, schlossen jedoch eine Jamaikakoalition aus Grünen, CDU und FDP ebenso wenig aus wie ein Bündnis mit der SPD und der Linkspartei.

Bereits einige Monate nach der Landtagswahl vom 5. September 2004 hatte sich die Ausgangssituation der tradierten Parteien im Saarland mit der Konstituierung einer neuen Partei links von der SPD unter der Führung Oskar Lafontaines geändert. Schon bei der

3 Vgl. zu den nach wie vor nicht behobenen Strukturproblemen der FDP insbesondere Jürgen $R$. Winkler, Strukturprobleme und Zukunftschancen der Freien Demokraten, in: Jahrbuch zur Liberalismus-Forschung, 10. Jg. (1998), S. $131-154$. 
Bundestagswahl im Herbst 2005 schockte diese mit 18,5 Prozent der Stimmen die saarländische SPD, die zugleich noch einmal derart dramatisch einbrach, dass sie kaum Aussichten hatte, in absehbarer Zeit an die Macht zurückkehren zu können. Getragen wurde die neue Linkspartei von ehemaligen Wählern der SPD, die sich aus Enttäuschung über die Regierungspolitik Schröders von der Partei abgewendet hatten. Gemeinsam mit Lafontaine war Maas noch bei der Landtagswahl 2004 als Kritiker der Berliner Reformpolitik hervorgetreten, dann aber auf Distanz gegangen, nachdem Lafontaine ankündigt hatte, für den Fall, dass Schröder seinen Kurs nicht ändere, eine neue Linkspartei zu unterstützen. In keinem anderen westlichen Bundesland war deren Ausgangslage bei einer Landtagswahl denn auch derart gut ${ }^{4}$. Sie war sich eines beträchtlichen Wählerkontingents sicher und konnte sich auf einen in Teilen der Bevölkerung nach wie vor populären Frontmann stützen. Vor die Wahl gestellt, Müller oder Lafontaine zum Ministerpräsidenten zu wählen, sprachen sich im Vorfeld der Landtagswahl 2009 lediglich 58 Prozent für den amtierenden Regierungschef aus. Ein Drittel der Saarländer bevorzugten dagegen Lafontaine, auch 40 Prozent der SPD-Anhänger 5 .

Neben den genannten Faktoren bestimmen sozialstrukturelle und konjunkturelle Rahmenbedingungen die Ausgangssituation der Parteien bei Wahlen ${ }^{6}$. Problematische gesellschaftliche und ökonomische Entwicklungen fordern politische Akteure heraus. Ob diese frühzeitig identifiziert und analysiert werden, ob Handlungsoptionen ausgelotet und akzeptable Lösungen zur Behebung von Strukturproblemen vorgeschlagen werden, beeinflusst die Wahrnehmung der Parteikompetenz und die Bewertung der Regierungsleistung.

Seit Jahrzehnten leidet das Saarland wie kein anderes altes Bundesland unter einem ökonomischen und technischen Strukturwandel. Wegen großer Probleme vor allem in den das Saarland traditionell prägenden Wirtschaftszweigen Stahlerzeugung und Bergbau und einer dadurch bedingten überdurchschnittlich hohen Arbeitslosenquote zählt es zu den größten Krisenregionen Deutschlands. Entsprechend negativ schätzen die Bürger seit zwei Jahrzehnten die wirtschaftliche Lage ihres Landes ein. Im Vorfeld der Landtagswahl meinten nur neun von 100 Wahlberechtigten, die wirtschaftliche Lage sei gut, dagegen glaubten 36 Prozent, sie sei schlecht. Deutlich negativer als 2004 fiel bei den Bürgern der Vergleich aus: So meinte 2004 jeder Dritte, das Saarland sei schlechter als die anderen Bundesländer aufgestellt, 2009 war es jeder Zweite 7 . Die Wahrnehmung der Saarländer wich damit erheblich von der des amtierenden Ministerpräsidenten ab. Auch meinten etwas weniger als noch 2004, dass die eigene wirtschaftliche Lage gut sei. Nur 14 von 100 Saarländern glaubten, sie befänden sich in einer ökonomisch schlechten Situation. Wie schon im Vorfeld der vorangegangenen Landtagswahl erwartete auch 2009 nur jeder vierte Wahlberechtigte einen Aufschwung.

4 Vgl. zu den Chancen der Linkspartei Claudia Lau, Die Chancen der Linkspartei, Wiesbaden 2008 .

5 Vgl. Forschungsgruppe Wahlen e.V., Wahl im Saarland. Eine Analyse der Landtagswahl vom 30. August 2009, Mannheim 2009, S. 22.

6 Vgl. zur Erklärung des Wählerverhaltens jetzt vor allem Harald Schoen, Soziologische Ansätze in der empirischen Wahlforschung, in: Jürgen W. Falter / ders. (Hrsg.), Handbuch Wahlforschung, Wiesbaden 2005, S. 135 - 185; Harald Schoen / Cornelia Weins, Der sozialpsychologische Ansatz zur Erklärung von Wahlverhalten, in: ebenda, S. $187-242$.

7 Vgl. zur Einschätzung der wirtschaftlichen Lage im Saarland Forschungsgruppe Wahlen e.V., a.a.O. (Fn. 5), S. 26 f. 
Die Wählerrekrutierung der CDU wird im Saarland ferner von zwei strukturellen Bedingungen begünstigt. Obwohl das Land eine überdurchschnittliche Bevölkerungsdichte aufweist, ist es mit Ausnahme von Saarbrücken und Neunkirchen ländlich und kleinstädtisch geprägt. Hinzu kommt, dass das Saarland den höchsten Katholikenanteil aller Bundesländer aufweist. Beide Eigenschaften hemmen einerseits die Ausbreitung der SPD und der Grünen. Andererseits gibt es einen überdurchschnittlich hohen Arbeiteranteil, so dass cross-pressures an der Saar traditionell eine größere Rolle spielen als anderswo. Unabhängig davon schmälert der Wandel der Berufsstruktur die Chancen der SPD. Während Anfang der 1960er Jahre noch mehr als die Hälfte aller Erwerbstätigen Arbeiter waren, ist es derzeit nur noch jeder dritte. Dagegen hat sich der Prozentsatz der Angestellten und Beamten von 27 auf 54 Prozent verdoppelt. Da die bereits im 19. Jahrhundert herausgebildeten Koalitionen zwischen sozialen Großgruppen und politischen Parteien auf geringerem Niveau nach wie vor bestehen ${ }^{8}$, haben sich die Erfolgsbedingungen der Sozialdemokraten zunehmend verschlechtert, die der Christ- und Freidemokraten dagegen verbessert. Vor diesem Hintergrund war die 14-jährige Regierungszeit der Sozialdemokraten in den 1980er und 1990er Jahren Ergebnis kurzfristig wirkender Einflussfaktoren des Wählerverhaltens wie der positiven Wahrnehmung Oskar Lafontaines und der Problemlösungskompetenz der SPD.

\section{Der Wahlkampf}

Schon im Januar 2009 waren sich die politischen Beobachter darin einig, dass die CDU zwar die absolute Mehrheit verlieren, aber dennoch wieder als stärkste Partei in den Landtag einziehen würde. Folgerichtig bestand das primäre Ziel der Christdemokraten darin, zusammen mit der FDP die Mehrheit der Sitze zu gewinnen. Peter Müller trat erneut als Spitzenkandidat der Union an. 2004 war die CDU noch gut beraten, ihn wegen seiner damaligen relativen Popularität in den Mittelpunkt ihres Wahlkampfes zu stellen. Da seine Beliebtheit jedoch deutlich nachgelassen hatte und ihm weniger positive Eigenschaften als noch 2004 zugeschrieben wurden, war die Strategie diesmal verfehlt, ihn als Landesvater und Integrationsfigur in den Vordergrund zu schieben. Angesichts der gescheiterten Wahl von Andrea Ypsilanti zur hessischen Ministerpräsidentin mit den Stimmen der Linkspartei rückte daher mehr und mehr die Koalitionsfrage ins Zentrum des CDU-Wahlkampfes. Die von ihr angestoßene, sich dem klassischen Freund-Feind-Schema bedienende Medienkampagne malte eine „rote Gefahr“ an die Wand. Während die CDU „Freiheit, Gerechtigkeit und soziale Verantwortung“ vertrete, stünden SPD und Linkspartei für „Gleichmacherei, staatlichen Umverteilungswahn und zentralistischen Dirigismus“. Die CDU müsse verhindern, dass die „Regierungsmarionette Heiko Maas“ vom „Strippenzieher“ Lafontaine eingesetzt werde: „Wer Maas wählt, kriegt Lafontaine." Jahren eine erfolgreiche Politik betrieben und das Land voran gebracht. Als Alternative zu einer von Müller geführten Regierung biete sich nur eine rot-rote Koalition an, die das Saarland ins Abseits steuerte. Sollte die CDU die Gelegenheit haben, weiter zu regieren,

8 Vgl. hierzu insbesondere Seymour Martin Lipset / Stein Rokkan, Cleavage Structures, Party Systems and Voter Alignments: An Introduction, in: dies. (Hrsg.), Party Systems and Voter Alignments, New York 1967, S. 1 - 64.

9 Peter Müller im Interview mit der Saarbrücker Zeitung vom 8. August 2009. 
würden Ausbildungsplätze für alle Jugendlichen bereitgestellt werden und alle älteren Arbeitslosen einen Arbeitsplatz finden. Außerdem sollte mehr für Bildung ausgegeben werden. Als Zugeständnis an die Grünen kann man den Hinweis verstehen, eventuell die Investitionen in erneuerbare Energien zu erhöhen.

Im letzten Wahlkampf hatte die Union der SPD angelastet, nicht genügend für die wirtschaftliche Entwicklung getan zu haben. Peter Müller hatte versprochen, das Saarland aus der Krise herauszuführen. Daran anknüpfend war es ein Teilziel der Union, im Land Optimismus zu verbreiten und die positiven Errungenschaften der vergangenen Jahre der Regierung Müller zuzuschreiben. Während die Sozialdemokraten die Region heruntergewirtschaftet hätten, habe Müller und sein Kabinett schwer daran gearbeitet, das Saarland wieder aufzurichten. Sie seien auf dem Gebiet der Wirtschaftspolitik derart erfolgreich, dass das Saarland schon bald wettbewerbsfähiger als die anderen Bundesländer sei. Vom Sorgenkind der Republik habe es sich zu einem der dynamischsten Bundesländer entwickelt ${ }^{10}$.

Wie schon bei den beiden vorausgegangen Landtagswahlen blieb auch diesmal die Arbeitslosigkeit das mit weitem Abstand wichtigste Problem. Für 60 Prozent aller Saarländer war dies das zentrale politische Thema, jeder fünfte rechnete auch die Schul- und Bildungspolitik dazu. Im Vergleich zu früheren Wahlen spielte die Kohle- und Stahlindustrie diesmal eine vergleichsweise geringe Rolle ${ }^{11}$. Ausschlaggebend für die Wahlentscheidung ist aber nicht nur, welche Themen im Wahlkampf auf der politischen Agenda stehen. Von noch größerem Einfluss ist die wahrgenommene Kompetenz der Parteien und Kandidaten. Deshalb konzentrierte sich die Union vor allem darauf, ihre Fähigkeiten und die ihres Spitzenkandidaten auf dem Gebiet der Wirtschafts- und Arbeitsmarktpolitik zu unterstreichen und der SPD sowie ihrem Spitzenkandidaten jegliche Kompetenz zur Lösung der Strukturprobleme abzusprechen. Die Saarländer hatten allerdings eine andere Wahrnehmung. Der Regierungspartei wurde deutlich geringere Problemlösungskompetenz als noch 2004 zugeschrieben, und zwar auch bei der Bekämpfung der Arbeitslosigkeit. Zwar schrieben die Bürger der CDU in der Wirtschafts- und Arbeitsmarktpolitik nach wie vor größere Fähigkeiten zu, auf dem Gebiet der Bildungspolitik lag sie jedoch deutlich hinter der SPD ${ }^{12}$.

Da die Sozialdemokraten wegen der Kandidatur der Linkspartei keinerlei Aussicht hatten, zusammen mit den Grünen eine Mehrheit im Landtag zu erhalten, und die CDU die Koalitionsfrage ins Zentrum der politischen Diskussion stellte, musste die SPD einen inhaltlichen Wahlkampf führen, um ihre Problemlösungskompetenz aufzeigen zu können. Begünstigt wurde eine derartige Strategie von den sozialen und wirtschaftlichen Rahmenbedingungen im Saarland, den ungünstigen Popularitätswerten des amtierenden Ministerpräsidenten sowie den sich aufhellenden Werten für die SPD und ihren Spitzenkandidaten Maas. Das Wahlkampfziel bestand darin, die CDU als führende Regierungspartei abzulösen. Wie dies angesichts der äußeren Rahmenbedingungen und der von allen Konkurrenten formulierten Bedingungen möglich sein sollte, war den politischen Beobachtern stets rätselhaft. Da eine Große Koalition mit dem Wahlziel unvereinbar und eine Ampel aus SPD, Grünen und FDP unrealistisch war, blieb einzig eine Koalition aus SPD, Grünen

10 Vgl. die „Leitlinien der CDU Saar zur Landtagswahl am 30. August 2009“, beschlossen vom 58. CDU-Landesparteitag am 19. Juni 2009, http://www.cdu-saar.de/downloads/87003.pdf (Abruf am 10. Mai 2010).

11 Vgl. Forschungsgruppe Wahlen e.V., a.a.O. (Fn. 5), S. 25 f.

12 Vgl. ebenda. 
und Linkspartei als Alternativregierung im Saarland übrig. Wegen der negativen Erfahrungen in Hessen wagte sich die SPD Saar allerdings nicht, das angestrebte Bündnis mit der Linkspartei auch offensiv zu vertreten. Die kategorische Ablehnung Lafontaines als Ministerpräsidenten selbst dann, wenn die Linkspartei stärker als die SPD aus der Wahl hervorgehen sollte, dürfte einige Dissonanzen bei SPD-Anhängern hervorgerufen haben, die sich Lafontaine als neuen Regierungschef vorstellen konnten. Zur Wahlkampfstrategie der SPD gehörte es folglich, dem amtierenden Kabinett Müller Leistungen auf allen wichtigen Politikfeldern abzusprechen und zugleich Reformperspektiven aufzuzeigen. In ihrem Regierungsprogramm prangerte sie die Versäumnisse der CDU im Land an. Sie plädierte für eine „aktive Wirtschafts- und Strukturpolitik“. Der Bergbau im Saarland sollte erhalten werden, mittelständische Existenzgründungen und wirtschaftsnahe Forschung gefördert, die Verwaltung grundlegend reformiert, die Studiengebühren abgeschafft und die Gymnasien in Ganztagsschulen integriert werden ${ }^{13}$.

Das primäre Ziel der Grünen war der Wiedereinzug in den Landtag. Für den Fall ihrer Regierungsbeteiligung strebten sie einen Politikwechsel in einigen Kernbereichen an. Als ihren Spitzenkandidaten präsentierten sie wie schon 2004 Hubert Ulrich. In ihrem stärker inhaltlich geführten Wahlkampf sprachen die Grünen vor allem die Themen Klima, Umwelt, Bildung und Gerechtigkeit an. Sie forderten ein gerechteres Bildungssystem, das allen Schülern die gleichen Chancen bietet. Ferner sollte eine nachhaltigere, sich stärker auf dezentrale Einheiten stützende und den Ausbau erneuerbarer Energien fördernde Energiepolitik betrieben werden ${ }^{14}$. Als Bedingung für eine Koalition mit der SPD formulierten die Grünen den endgültigen und sofortigen Ausstieg aus der Steinkohleförderung. Um sich alle Koalitionstüren offen zu lassen, gingen sie zwar ohne formelle Koalitionsaussage in den Wahlkampf, liebäugelten aber mit einer unrealistischen Ampel aus SPD, Grünen und FDP. Weder eine Jamaikakoalition aus CDU, FDP und Grünen noch eine rot-rot-grüne Koalition wurde ausgeschlossen unter der Bedingung, dass der Ministerpräsident nicht Lafontaine heiße und der Steinkohlebergbau eingestellt werde.

Da die FDP diesmal davon ausgehen konnte, den Einzug in das Landesparlament zu schaffen, strebte sie ebenso wie in den meisten anderen Bundesländern nunmehr eine Regierungsbeteiligung an. Angesichts der bundespolitischen Konstellationen hatten sich die Freidemokraten schon früh auf eine Koalition mit der CDU festgelegt und andere Optionen ausgeschlossen. Um Koalitionswähler zu gewinnen, sprach die FDP deshalb auch frühere CDU-Wähler an. Im Einklang mit der programmatisch-ideologischen Ausrichtung der Bundespartei versprach sie „mehr Netto vom Brutto“, eine Senkung der Gewerbesteuer, die Abschaffung der Vermögenssteuer und niedrigere Abgaben nach Einführung eines „einfachen“ Drei-Stufen-Steuermodells. Die öffentlichen Ausgaben sollten dafür heruntergefahren werden ${ }^{15}$. Ferner sollte der Steinkohlebergbau schnellstmöglichst beendet, das

13 Vgl. das Wahlprogramm der SPD zur Landtagswahl 2009, „Regierungsprogramm für ein faires, modernes Saarland“, beschlossen auf dem 26. Ordentlichen SPD-Landesparteitag am 8./9. Mai 2009, http://www.spd-saar.de/fileadmin/wahl2009/Materialien/Landtagswahl/LPT09_Regierungsprogramm_mitCOVER.pdf (Abruf am 10. Mai 2010).

14 Vgl. auch das Landtagswahlprogramm 2009 von Bündnis90/Die Grünen Saar, „Zeit für Veränderung", http://www.gruene-saar.de/uploads/PDF/Lwp2009.pdf (Abruf am 10. Mai 2010).

15 Vgl. das Landtagswahlprogramm 2009 der FDP, „Programm für dich“, http://www.fdpsaar.de/ images/cms/download/files/FDP-Saar_Landtagswahlprogramm_2009.pdf (Abruf am 10. Mai 2010). 
Ladenschlussgesetz für Werktage abgeschafft und das Saarland zur Modellregion für Existenzgründer gemacht werden.

Der Erfolg der Linkspartei bei der Bundestagswahl 2005 hatte ihre Entwicklung an der Saar beflügelt und die Messlatte für die Landtagswahl aufgelegt. Wenngleich die Partei vorgab, Lafontaine erneut zum saarländischen Ministerpräsidenten machen zu wollen, bestand das eigentliche Wahlziel doch darin, die 20-Prozent-Marke zu überspringen, um so Druck vor allem auf die SPD im Land und im Bund ausüben zu können. An der Saar sollte der Beweis erbracht werden, dass das gute Abschneiden der Linkspartei bei der Bundestagswahl 2005 kein Betriebsunfall der SPD war. Aufgrund des Bekanntheitsgrades und der Popularität Lafontaines im linken Wählerlager, das die Partei gezielt anzusprechen versuchte, führte sie einen inhaltlich unterfütterten Personenwahlkampf. Einerseits rückte sie ihren Spitzenkandidaten als Symbolfigur der Linken in den Vordergrund. Andererseits agitierte die Linke gegen die sozialpolitischen Maßnahmen der früheren Regierung Schröder, die zur Abwanderung zahlreicher Wähler und Mitglieder von der SPD geführt hatten. Die Strategie konnte überzeugen, weil ihr Spitzenkandidat für genau diese Ausrichtung stand. Zur Personalisierungsstrategie gehörte neben der Personenplakatierung die Darstellung der Lebensabschnitte Lafontaines vom Schüler bis zum Spitzenkandidaten der Linken unter www. oskar-waehlen.de, zum Themenwahlkampf die Fokussierung auf zentrale Felder, insbesondere die Sozialpolitik. So forderte die Linkspartei einen grundlegenden Politikwechsel ${ }^{16}$, indem unter anderem Reichtum besser verteilt, die Lebensqualität und Chancen der Benachteiligten erhöht, Hartz IV, die Rente mit 67 und die Studiengebühren abgeschafft werden sollten. Falls sie an die Regierung komme, werde ein Sozialpass eingeführt werden, der es den Menschen mit geringen Einkommen ermögliche, Freizeit- und Kultureinrichtungen zu besuchen und den öffentlichen Personennahverkehr kostenlos oder preisermäBigt zu nutzen.

\section{Das Wahlergebnis}

Von den 804.622 Wahlberechtigten im Saarland gingen am 30. August 2010544.220 zur Wahl ${ }^{17}$. Gegenüber der Landtagswahl 2004, bei der so wenige Bürger wie nie zuvor die Wahllokale aufgesucht hatten, stieg die Wahlbeteiligung um 12,1 Punkte auf 67,6 Prozent und erreichte damit in etwa das Niveau der letzten Wahl im 20. Jahrhundert. Am geringsten war die Beteiligung auch diesmal wieder in den größeren Städten: Neunkirchen, Völklingen, Dillingen, Homburg und Saarbrücken. Der deutliche Anstieg in allen Gemeinden - er variierte zwischen 8,2 (Großrosseln) und 18,1 Punkten (Wallerfangen) - dokumentiert, dass es den Parteien diesmal besser gelang, die Bürger zu mobilisieren.

Die beiden Großparteien sind die Verlierer der Wahl. Die CDU büßte gegenüber der Landtagswahl 200413 Punkte ein und erzielte mit 34,5 Prozent der Stimmen das schlechteste Ergebnis bei einer Landtagswahl im Saarland seit dem Jahr der deutschen Vereini-

16 Vgl. das Landtagswahlprogramm 2009 der Linkspartei, „Dafür steht die Linke in unserem Saarland“, http://www.dielinke-saar.de/fileadmin/Pdf-Dateien/Dokumente/Wahlbroschuere_Internet.pdf (Abruf am 10. Mai 2010).

17 Statistisches Amt Saarland (Hrsg.), Landtagswahl am 30. August 2009. Vorläufige Ergebnisse, Saarbrücken 2009. 


\begin{tabular}{|c|c|c|c|c|c|c|c|c|c|}
\hline \multicolumn{10}{|c|}{$\begin{array}{l}\text { Tabelle 1: Stimmenanteile der Parteien im Saarland } 1994 \text { bis } 2009 \text { (Angaben in Prozent der } \\
\text { abgegebenen gültigen Stimmen) }\end{array}$} \\
\hline Partei & $\begin{array}{l}\text { LTW } \\
1994\end{array}$ & $\begin{array}{l}\text { BTW } \\
1994\end{array}$ & $\begin{array}{l}\text { BTW } \\
1998\end{array}$ & $\begin{array}{l}\text { LTW } \\
1999\end{array}$ & $\begin{array}{l}\text { BTW } \\
2002\end{array}$ & $\begin{array}{l}\text { LTW } \\
2004\end{array}$ & $\begin{array}{l}\text { BTW } \\
2005\end{array}$ & $\begin{array}{l}\text { LTW } \\
2009\end{array}$ & $\begin{array}{l}\text { BTW } \\
2009\end{array}$ \\
\hline SPD & 49,4 & 48,8 & 52,4 & 44,4 & 46,0 & 30,8 & 33,3 & 5 & 24,7 \\
\hline $\mathrm{CDU}$ & 38,6 & 37,2 & 31,8 & 45,5 & 35,0 & 47,5 & 30,2 & 34,5 & 30,7 \\
\hline FDP & 2,1 & 4,3 & 4,7 & 2,6 & 6,4 & 5,2 & 7,4 & 9,2 & 11,9 \\
\hline Grüne & 5,5 & 5,8 & 5,5 & 3,2 & 7,6 & 5 , & 5,9 & 5,9 & 6,8 \\
\hline Die Linke* & - & 0,7 & 1,0 & 0,8 & 1,4 & 2,3 & 18,5 & 21,3 & 21,2 \\
\hline Sonstige & 4,5 & 3,2 & 4,5 & 3,5 & 3,7 & 8,6 & 4,7 & 4,6 & 4,8 \\
\hline Wahlbeteiligung & 83,5 & 83,5 & 84,8 & 68,7 & 80,0 & 55,5 & 79,4 & 67,6 & 73,7 \\
\hline \multicolumn{10}{|c|}{$\begin{array}{l}\text { LTW: Landtagswahl; BTW: Bundestagswahl. } \\
\text { * Vor } 2005 \text { PDS. } \\
\text { Quelle: Statistisches Amt Saarland, Landtagswahlen } 1947 \text { bis } 2004 \text { im Saarland; dass., Bundestagswah- } \\
\text { len } 1957 \text { bis } 2005 \text { im Saarland; dass., Endgültiges Ergebnis der Bundestagswahl 2009; dass., Endgültiges } \\
\text { amtliches Endergebnis der Landtagswahl } 2009 \text {. }\end{array}$} \\
\hline
\end{tabular}

gung. Noch nie zuvor hatten die Christdemokraten in einem westdeutschen Flächenland derart große Verluste bei einer Landtagswahl in Kauf nehmen müssen. Dennoch lagen sie deutlich über dem Resultat der Bundestagswahl 2005. Nachdem die CDU bei den beiden vorangegangenen Landtagswahlen jeweils die absolute Mehrheit der Mandate errungen hatte, blieb sie diesmal mit nur noch 19 von 51 Sitzen deutlich unter der erforderlichen Zahl von 26 Mandaten. Nach zehn Jahren verlor sie damit die absolute Mehrheit im Saarländischen Landtag. Anders als 2004 erhielt Peter Müller zwar kein Votum für die Fortführung der Regierungsarbeit, doch angesichts des sehr schwachen Abschneidens der SPD blieb die CDU dominante Partei, zu der sie 1994 aufgestiegen war. Ihre besten Resultate erzielte die CDU in Tholey (47,5 Prozent), Eppelborn (46,5 Prozent), Perl (46,3 Prozent) und Oberthal (44,3 Prozent). Während sie in Nalbach 5,1 Punkte hinzugewinnen konnte, musste sie in zahlreichen Gemeinden dramatische Verluste verkraften, die höchsten in Weiskirchen (-17,9), Mettlach (-17,7), Losheim am See (-17,2), Freisen $(-16,5)$ und Nonnweiler $(-16,5)$. Zwar gewann die CDU einige Nichtwähler des Jahres 2004 zurück, doch konnten diese die Abwanderungen insbesondere zur FDP und ins Nichtwählerlager, in kleineren Teilen auch zu SPD und Linkspartei nicht ausgleichen.

Auch die SPD erreichte ihr Wahlziel nicht. Sie verlor gegenüber 2004 noch einmal 6,3 Punkte und erzielte mit nur noch 24,5 Prozent der Stimmen das schlechteste Ergebnis seit der Eingliederung des Saarlandes in die Bundesrepublik. In nur zehn Jahren sank ihr Stimmenanteil um fast 20 Prozentpunkte. Im Vergleich zur Landtagswahl 1994 hat er sich sogar halbiert. Einen derart dramatischen Niedergang einer Partei hat es bei Landtagswahlen in der Bundesrepublik noch nie gegeben. Wenngleich die SPD einige CDU-Wähler und auch einen kleinen Anteil der Nichtwähler des Jahres 2004 zurückgewinnen konnte, reichte dies nicht aus, um die Abwanderungen zu kompensieren ${ }^{18}$. Auch im Vergleich zur Bundestags-

18 Nach den Berechnungen von Infratest dimap für die ARD verlor die SPD 2004 rund 62.000 Wähler an die Partei der Nichtwähler. Davon wanderten 200914.000 zur SPD zurück. Vgl. Infratest dimap, Landtagswahl Saarland am 5. September 2004. Bericht von Infratest dimap für die ARD; dies., Landtagswahl Saarland am 30. August 2009. Bericht von Infratest dimap für die ARD. 
wahl 2005 büßte die SPD 8,8 Punkte ein. Angesichts der im Mittel etwas stärkeren Verluste in den früheren Hochburgen haben sich die Unterschiede in der regionalen Wählerrekrutierung auch bei der SPD etwas nivelliert. Ihr Stimmenanteil reicht nun von 15,4 Prozent in der Unionshochburg Nalbach bis 33,9 Prozent in Rehlingen-Siersburg. Weit überdurchschnittlich verloren die Sozialdemokraten in Saarlouis $(-13,2)$, Bous $(-12,8)$, Ensdorf $(-12,7)$ und auch in ihrer Diaspora Nalbach $(-12,2)$. Nur in Perl $(2,0)$ und Nonnweiler $(1,7)$ gewann sie kleinere Anteile hinzu ${ }^{19}$.

FDP und Grüne erreichten ihre primären Wahlziele und zogen erneut in den Saarländischen Landtag ein. Die Grünen gewannen 0,3 Punkte hinzu und erzielten mit 5,9 Prozent der Stimmen das Ergebnis der Bundestagswahl 2005. Die FDP legte 4,0 Punkte zu und erreichte mit 9,2 Prozent der Stimmen das zweitbeste Resultat seit 1985. In Nonnweiler konnte sie 6,2 und in Saarwellingen sogar 8,0 Punkte hinzugewinnen. Ihr mit großem Abstand bestes Ergebnis war mit 18,6 Prozent in Nalbach zu verzeichnen. Darüber hinaus erzielten die Freidemokraten in Saarwellingen (14,0 Prozent) und in Lebach (12,5 Prozent) beachtliche Ergebnisse. In Freisen und Großrosseln blieben sie dagegen unter sechs Prozent. Die Zuwächse kamen vor allem aus zwei Gruppen: frühere Wähler der CDU und ehemalige Nichtwähler. Die Grünen gewannen ihre höchsten Anteile in Saarlouis (9,3 Prozent) und Saarbrücken (9,0 Prozent), ihre niedrigsten in Nonnweiler (3,1 Prozent) und Freisen (3,2 Prozent). Bemerkenswert ist, dass die regionale Wählerstruktur der Grünen im Vergleich zur Landtagswahl 2004 keine nennenswerten Verschiebungen aufweist. Und wie bei der letzten Wahl blieb die Partei auch diesmal in der Hälfte der 51 Gemeinden des Saarlandes unter der Fünfprozentmarke.

Die Linkspartei war der große Wahlsieger. Sie gewann 18,9 Punkte hinzu und kam mit 21,3 Prozent der Stimmen nahe an das Ergebnis der SPD heran. Damit erzielte sie das mit großem Abstand beste Ergebnis in einem alten Bundesland. Mit elf Mandaten zog die Linke erstmals ins Parlament eines westlichen Bundeslandes ein. In einigen Gemeinden des Saarlandes verhalfen ihr erdrutschartige Bewegungen zum Erfolg. In Völklingen gewann sie 27,0, in Wallerfangen 25,5 und in Großrosseln und Schiffweiler jeweils 24,8 Punkte hinzu. Nur in Perl und Nalbach blieben die Zuwächse unter 10 Punkten. Die höchsten Stimmenanteile erzielte die Linkspartei in Völklingen (29,6 Prozent), Wallerfangen (27,7 Prozent) und Schiffweiler (27,6 Prozent). Am schlechtesten schnitt sie in Perl (9,6 Prozent) und Nalbach (10,8 Prozent) ab. Folgt man den Wählerwanderungsanalysen von Infratest dimap, so kamen etwa 47 Prozent der neuen Wähler der Linkspartei aus dem Nichtwählerlager, rund 28 Prozent hatten danach 2004 SPD und etwa 10 Prozent CDU gewählt. Von den verbleibenden 4,5 Prozent der Stimmen entfielen zwei Prozent auf die Familien-Partei Deutschlands und 1,5 Prozent auf die NPD, die gegenüber der vorangegangenen Landtagswahl damit rund zwei Drittel ihrer Wähler verlor.

Bei den Relationen zwischen den Stimmenanteilen der Parteien in den 52 Gemeinden des Saarlandes 2004 und $2009^{20}$ zeigt sich Folgendes: (1) CDU und Grüne weisen die höchste regionale Stabilität auf. Da diese bei der SPD deutlich zurückgegangen und bei der FDP stark angestiegen ist, zeigen beide Parteien im Saarland gegenwärtig eine ähnliche regionale Stabilität. Alle Parteien waren am 30. August 2009 dort überdurchschnittlich erfolg-

19 Die Ergebnisse in den Gemeinden finden sich unter http://www.statistikextern.saarland.de/wahlen/wahlen/2009/internet_saar/LT_SL_09/gemeindeergebnisse/ (Abruf am 10. Mai 2010).

20 Vgl. die Korrelationsmatrix in Forschungsgruppe Wahlen e.V., a.a.O. (Fn. 5), S. 88. 
reich, wo sie auch schon am 5. September 2004 gut abgeschnitten hatten; am stärksten gilt dies für CDU und Grüne. (2) Bei beiden Großparteien gibt es nach wie vor unterschiedliche regionale Schwerpunkte, die sich in einer starken negativen Korrelation der Stimmenanteile ausdrücken. (3) FDP und Grüne konkurrieren wieder etwas mehr um strukturverwandte Wählersegmente, als dies 2004 der Fall war. (4) Die Linkspartei bildet bezüglich der regionalen Wählerstruktur einen Gegenpol zur CDU. Sie erzielte bei der Landtagswahl 2009 dort überdurchschnittliche Resultate, wo 2004 die SPD besonders erfolgreich war. (5) Die CDU profitiert auch diesmal wieder von einer hohen Wahlbeteiligung in den Gemeinden. Je mehr Bürger partizipieren, desto höher fallen die Ergebnisse der Union und desto niedriger die Resultate von Grünen und SPD und insbesondere der Linkspartei aus.

\section{Das Wahlverhalten sozialer Gruppen}

\subsection{Wahlverhalten nach Geschlecht und Alter}

In den 1970er Jahren hatten Frauen im Saarland deutlich stärker die CDU und Männer mehr die SPD unterstützt. Im folgenden Jahrzehnt wandten sich Frauen verstärkt von der Union ab und der SPD zu, so dass mit Anfang der 1990er Jahre schließlich nur noch geringe Unterschiede im Wahlverhalten von Frauen und Männern beobachtet werden konnten $^{21}$. Auch bei der Landtagswahl 2009 tendierten Frauen etwas mehr zur Union. Anders als bei früheren Landtagswahlen wurde diesmal aber auch die SPD etwas stärker von Frauen als von Männern unterstützt. Dagegen neigten Männer deutlich öfter zur Linkspartei als Frauen. Damit bestätigte sich erneut, dass Männer eher als Frauen zur Unterstützung von Parteien rechts von der Union und links von der SPD tendieren.

Während sich das geschlechtsspezifische Wahlverhalten in den 1970er und 1980er Jahren angeglichen hatte, war das der jüngeren Wähler merklich von dem der älteren Bürger abgewichen. Weil die CDU bei den Jungwählern Stimmenanteile verlor, konnten die Sozialdemokraten für einige Jahre zur dominanten Partei im Saarland aufsteigen. Vor allem junge Frauen hatten sich dann Anfang der 1990er Jahre von der Union ab- und der SPD sowie den Grünen zugewandt. Erst 1999 konnte die CDU diesen Trend brechen und schließlich sogar umkehren. Jüngere Bürger verhalfen Müller an die Regierung. Die einsetzende Abwanderung junger Wähler veränderte so das Altersprofil von SPD und Grünen merklich ${ }^{22}$. Die Wahlchancen der Sozialdemokraten an der Saar wurden dadurch beträchtlich gemindert.

Wie schon 2004 wurde die CDU wieder in allen Altersgruppen stärkste Partei. Am meisten neigen nach wie vor die über 60 Jahre alten Frauen zur Union. Die SPD verlor erneut in allen Altersgruppen Stimmenanteile. Das Alter hatte dennoch keinen Einfluss auf

21 Vgl. zum geschlechtsspezifischen Wahlverhalten in der Bundesrepublik Deutschland vor allem Jürgen W. Falter / Siegfried Schumann, Vive la (très) petite différence! Über das unterschiedliche Wahlverhalten von Männern und Frauen bei der Bundestagswahl 1987, in: Max Kaase / HansDieter Klingemann (Hrsg.), Wahlen und Wähler. Analysen aus Anlaß der Bundestagswahl 1987, Opladen 1990, S. 109 - 142; Ute Molitor, Wählen Frauen anders? Zur Soziologie eines frauenspezifischen politischen Verhaltens in der Bundesrepublik Deutschland, Baden-Baden 1992.

22 Vgl. zum wandelnden Profil der Grünen auch Markus Klein / Kai Arzheimer, Grau in Grau. Die Grünen und ihre Wähler nach eineinhalb Jahrzehnten, in: Kölner Zeitschrift für Soziologie und Sozialpsychologie, 49. Jg. (1997), H. 4, S. $650-673$. 
ihre Wahl. Während jüngere Wähler besonders stark zu den Grünen tendierten, gingen die über 60 Jahre alten Saarländer auf eine besonders große Distanz zu ihnen ${ }^{23}$. Im Gegensatz zu den Grünen konnte die FDP in allen Altersgruppen ähnlich große Kontingente gewinnen. Die Linkspartei war besonders bei den Wählern mittleren Alters erfolgreich.

\subsection{Wahlverhalten nach Konfessionszugehörigkeit}

Seit dem 19. Jahrhundert hat die konfessionelle Zusammensetzung der Regionen die Struktur der Parteiensysteme in hohem Maße bestimmt, da Katholiken traditionell stärker zu christlich orientierten Parteien tendieren. Die Konfessionsstruktur einer Region ist eine der wichtigsten Rahmenbedingungen des Wahlverhaltens ${ }^{24}$. Dass mehr als zwei Drittel der Saarländer der katholischen Kirche angehören, begünstigt seit Jahrzehnten die Wählerrekrutierung der CDU. Im August 2009 verlor die Union unabhängig von der Konfessionszugehörigkeit Stimmenanteile und kam bei den Katholiken noch auf eine Zustimmung von 40 Prozent. Durch die hohen Verluste fiel sie sowohl in der Gruppe der Protestanten als auch in der der Konfessionslosen hinter die Stimmenanteile der SPD zurück, bei den Konfessionslosen auch hinter die der Linkspartei. Die SPD büßte ebenfalls Stimmen in allen konfessionellen Gruppen ein, am meisten unter den Protestanten und Konfessionslosen. In der Gruppe der Protestanten blieb sie allerdings trotz der überdurchschnittlichen Verluste stärkste politische Kraft. Die wachsende Zahl der konfessionslosen Bürger tendierte überdurchschnittlich zu den Grünen, der FDP und der Linkspartei. Jeder dritte konfessionslose Wähler stimmte bei der Landtagswahl am 30. August 2009 für die Linkspartei.

\subsection{Wahlverhalten nach Berufstätigkeit}

Seit dem späten 19. Jahrhundert prägt eine Koalition zwischen Arbeitern und der SPD die Struktur des deutschen Parteiensystems ${ }^{25}$. Die lange Jahre stetig steigende Anzahl der Arbeiter hatte den Aufstieg der Sozialdemokratie auch im Saarland maßgeblich bestimmt. Mit dem Übergang von der Industrie- zur Dienstleistungsgesellschaft sank der Arbeiter-

23 Vgl. zum Wahlverhalten nach Geschlecht und Alter Forschungsgruppe Wahlen e.V., a.a.O. (Fn. 5), S. $30 \mathrm{f}$.

24 Vgl. hierzu Jürgen R. Winkler, Sozialstruktur, politische Traditionen und Liberalismus. Eine empirische Längsschnittstudie zur Wahlentwicklung in Deutschland 1871-1933, Opladen 1995; Karl Schmitt, Konfession und Wahlverhalten in der Bundesrepublik Deutschland, Berlin 1989; Franz Urban Pappi, Die konfessionell-religiöse Konfliktlinie in der deutschen Wählerschaft. Entstehung, Stabilität und Wandel, in: Dieter Oberndörfer / Hans Rattinger / Karl Schmitt (Hrsg.), Wirtschaftlicher Wandel, religiöser Wandel und Wertwandel. Folgen für das politische Verhalten in der Bundesrepublik Deutschland, Berlin 1985, S. 263 - 290.

25 Vgl. unter anderem Franz Urban Pappi, Das Wahlverhalten sozialer Gruppen bei Bundestagswahlen im Zeitvergleich, in: Hans-Dieter Klingemann / Max Kaase (Hrsg.), Wahlen und politischer Prozeß. Analysen aus Anlaß der Bundestagswahl 1983, Opladen 1986, S. 369 - 394. Harald Schoen / Jürgen W. Falter, Wahlen und Wählerverhalten, in: Thomas Ellwein / Everhard Holtmann (Hrsg.), 50 Jahre Bundesrepublik Deutschland. Rahmenbedingungen - Entwicklungen - Perspektiven, Opladen 1999, S. $454-470$. 


\begin{tabular}{|c|c|c|c|c|c|c|c|c|c|c|c|c|c|}
\hline \multicolumn{14}{|c|}{$\begin{array}{l}\text { Tabelle 2: Wablverhalten nach der Konfessionszugehörigkeit bei Wablen zum Saarländischen } \\
\text { Landtag } 1999 \text { bis } 2009 \text { (Angaben in Prozent) }\end{array}$} \\
\hline \multirow[t]{2}{*}{ Konfession } & \multicolumn{3}{|c|}{ SPD } & \multicolumn{3}{|c|}{$\mathrm{CDU}$} & \multicolumn{3}{|c|}{ FDP } & \multicolumn{3}{|c|}{ Grüne } & \multirow{2}{*}{\begin{tabular}{|l|} 
Linke \\
2009
\end{tabular}} \\
\hline & 2009 & 2004 & 1999 & 009 & 2004 & 1999 & 2009 & 2004 & 1999 & 2009 & 2004 & 1999 & \\
\hline Kath & 23 & 28 & 42 & 40 & 53 & 50 & 9 & J & 2 & & 5 & 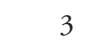 & 19 \\
\hline Evangelisch & 33 & 42 & 54 & 26 & 34 & 34 & 9 & 7 & 4 & 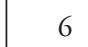 & 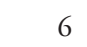 & 3 & 21 \\
\hline Keine & 21 & 32 & 41 & 17 & 30 & 35 & 13 & 8 & 4 & 12 & 15 & 11 & 32 \\
\hline Alle & 24,5 & 30,8 & 44,4 & 34,5 & 47,5 & 45,5 & 9,2 & 5,2 & 2,6 & 5,9 & 5,6 & 3,2 & 21,3 \\
\hline \multicolumn{14}{|c|}{$\begin{array}{l}\text { Quelle: Forschungsgruppe Wahlen e.V., Wahl im Saarland. Eine Analyse der Landtagswahl vom 5. Sep- } \\
\text { tember 1999, Mannheim 1999, S. 22; dies., Wahl im Saarland. Eine Analyse der Landtagswahl vom } \\
\text { 5. September 2004, Mannheim 2004, S. 49; dies., Wahl im Saarland. Eine Analyse der Landtagswahl } \\
\text { vom 30. August 2009, Mannheim 2009, S. } 34 \text {. }\end{array}$} \\
\hline
\end{tabular}

anteil an der erwerbstätigen Bevölkerung jedoch immer mehr. Zugleich stieg der Prozentsatz der Angestellten und Beamten kontinuierlich an. So ist der Arbeiteranteil im Saarland seit Anfang der 1960er Jahre auf nur noch ein Drittel der Erwerbstätigen geschrumpft. Auf gut über die Hälfte aller Erwerbstätigen hat sich dagegen der Anteil der Angestellten und Beamten verdoppelt. Die Zukunft der Parteien hängt daher heute davon ab, ob es ihnen gelingt, genügend Angehörige der so genannten neuen Mittelschicht der Beamten und Angestellten zu mobilisieren ${ }^{26}$.

Bei der Landtagswahl 2009 verlor die CDU vor allem Arbeitslose, Selbstständige und Angestellte unter ihren Wählern. Die stärkste Unterstützung erfuhr sie von den Beamten, gefolgt von den Selbstständigen und Rentnern, die geringste von den Arbeitslosen und Arbeitern. Jeder zweite Beamte, aber nur jeder vierte Arbeiter wählte diesmal die CDU. Bei den Angestellten, Beamten und Selbstständigen blieb sie stärkste politische Partei.

Die SPD verlor 2009 erneut in allen Berufsgruppen Stimmenanteile, am meisten bei den Rentnern und Arbeitern, die dennoch als einzige Gruppe etwas überdurchschnittlich zur SPD tendierten. Am geringsten war erneut die Neigung der Selbstständigen zur Wahl der SPD. Unterdurchschnittliche Anteile erzielte sie auch bei den Beamten und Arbeitslosen. Während 1999 noch jeder zweite Arbeitslose die Sozialdemokraten unterstützt hatte, wählte diesmal nur noch jeder Fünfte SPD. Hatten 1994 noch zwei von drei Arbeitern sich für die SPD entschieden, waren es 2009 nur noch 28 Prozent. Innerhalb von nur fünfzehn Jahren verlor die SPD Saar damit in dieser Berufsgruppe 36 Prozentpunkte. Diese Abwanderung hat dem Niedergang der SPD an der Saar einen kräftigen Schub versetzt. Nicht einmal jeder dritte SPD-Wähler gehört mittlerweile der Gruppe der Arbeiter an. Dagegen sind etwas über 40 Prozent als Angestellte tätig.

Die Linkspartei wurde bei den Arbeitern und den Arbeitslosen stärkste Partei. Fast jeder dritte Arbeiter und 44 Prozent der Arbeitslosen stimmten am 30. August 2009 für sie. Ihre Wahlkampfstrategie war damit aufgegangen. Weit unterdurchschnittlich neigten lediglich Beamte und Selbstständige zur Linkspartei.

26 Vgl. zu den Herausforderungen dieser Entwicklung für die Sozialdemokraten vor allem Herbert Kitschelt, The Transformation of Social Democracy in Western Europe, Cambridge 1994. 


\begin{tabular}{|c|c|c|c|c|c|c|c|c|c|c|c|c|c|}
\hline \multirow[t]{2}{*}{ Sozialmerkmal } & \multicolumn{3}{|c|}{ SPD } & \multicolumn{3}{|c|}{$\mathrm{CDU}$} & \multicolumn{3}{|c|}{ FDP } & \multicolumn{3}{|c|}{ Grüne } & \multirow{2}{*}{\begin{tabular}{|l|l} 
Linke \\
2009 \\
\end{tabular}} \\
\hline & 2009 & 2004 & 1999 & 2009 & 2004 & 1999 & 2009 & 2004 & 1999 & 2009 & 2004 & 1999 & \\
\hline Berufstätigkeit & & & & & & & & & & & & & \\
\hline $\begin{array}{l}\text { berutstätig } \\
\text { Rentner }\end{array}$ & $\begin{array}{l}25 \\
25\end{array}$ & $\begin{array}{l}30 \\
34\end{array}$ & $\begin{array}{l}45 \\
45\end{array}$ & $\begin{array}{l}31 \\
41\end{array}$ & $\begin{array}{l}45 \\
52\end{array}$ & $\begin{array}{l}44 \\
48\end{array}$ & $\begin{array}{r}10 \\
8\end{array}$ & $\begin{array}{l}6 \\
4\end{array}$ & $\begin{array}{l}3 \\
3\end{array}$ & $\begin{array}{l}7 \\
3\end{array}$ & $\begin{array}{l}7 \\
2\end{array}$ & $\begin{array}{l}4 \\
1\end{array}$ & $\begin{array}{l}22 \\
20\end{array}$ \\
\hline arbeitslos & 20 & 26 & 52 & 15 & 35 & 36 & 5 & 3 & 1 & 5 & 6 & 2 & 44 \\
\hline \multicolumn{14}{|l|}{ Beruf } \\
\hline Arbeiter & 28 & 38 & 56 & 26 & 37 & 35 & 6 & 3 & 1 & 3 & 3 & 2 & 31 \\
\hline Angestellte & 26 & 30 & 44 & 32 & 47 & 45 & 10 & 6 & 3 & 7 & 7 & 4 & 21 \\
\hline Beamte & 21 & 25 & 32 & 51 & 59 & 59 & 8 & 6 & 3 & 6 & 5 & 4 & 11 \\
\hline Selbstständige & 13 & 20 & 25 & 43 & 59 & 62 & 19 & 10 & 6 & 8 & 6 & 6 & 13 \\
\hline Alle & 24,5 & 30,8 & 44,4 & 34,5 & 47,5 & 45,5 & 9,2 & 5,2 & 2,6 & 5,2 & 5,6 & 3,2 & 21,3 \\
\hline
\end{tabular}

\subsection{Wahlverhalten nach Gewerkschaftszugehörigkeit}

Wegen der im katholischen Milieu vorherrschenden Wahlnorm hatte die SPD an der Saar lange Zeit nur einen vergleichsweise geringen Anteil der Gewerkschaftsmitglieder rekrutieren können. Deren konfessionelle Zusammensetzung begrenzte ihre Mobilisierungschancen. Als es der SPD jedoch gelang, die katholischen Gewerkschaftsmitglieder an sich zu binden, war der Aufstieg zur dominanten Partei im Saarland geebnet. Anfang der 1990er Jahre tendierten die Gewerkschaftler schließlich deutlich stärker als im Bundesgebiet zur Wahl der SPD. Die Klassenwahl prägte nun einige Jahre besonders stark die Struktur des saarländischen Wählerverhaltens. Schon 2004 hatten sich jedoch Abwanderungstendenzen der Gewerkschaftler von der SPD gezeigt. Viele waren mit der Politik der SPD im Bund unzufrieden und auf Distanz zu ihr gegangen.

Bei der Landtagswahl 2009 verlor die SPD in dieser Gruppe erneut elf Prozentpunkte. Vor allem die gewerkschaftlichen Arbeiter wandten sich von ihr ab. Etwa jeweils ein Drittel der Gewerkschaftsmitglieder stimmte diesmal für SPD und die Linkspartei. Die Krise der SPD zeigt sich besonders, wenn man zum Vergleich die Landtagswahl 1994 heranzieht, bei der noch fast zwei Drittel der Gewerkschaftsmitglieder die SPD gewählt hatten ${ }^{27}$.

27 Vgl. zum Wahlverhalten der Gewerkschaftsmitglieder bei den Landtagswahlen im Saarland Forschungsgruppe Wahlen e. V., Wahl im Saarland. Eine Analyse der Landtagswahl vom 16. Oktober 1994, Mannheim 1994, S. 15; Forschungsgruppe Wahlen e.V., Wahl im Saarland. Eine Analyse der Landtagswahl vom 5. September 1999, Mannheim 1999, S. 17; Forschungsgruppe Wahlen e. V., Wahl im Saarland. Eine Analyse der Landtagswahl vom 5. September 2004, Mannheim 2004, S. 43; Forschungsgruppe Wahlen e.V., Wahl im Saarland. Eine Analyse der Landtagswahl vom 30. August 2009, Mannheim 2009, S. 33. 


\section{Regierungs- und Oppositionsbildung}

Das Stimmverhalten der Saarländer strafte die beiden Großparteien ab, stärkte der oppositionellen FDP den Rücken und trug die von Lafontaine geführte Linkspartei erstmals in den Landtag. Eine Bestätigung der amtierenden Regierung unter Ministerpräsident Müller war es nicht. Die Union verlor acht Mandate und verfügt nur noch über 19 der 51 Sitze im Landtag. Die SPD hält statt zuvor 18 nur noch 13 Mandate. Die Grünen behalten ihre drei Sitze, die FDP gewinnt zwei hinzu und ist nun mit fünf Abgeordneten vertreten. Die Linkspartei stellt elf Parlamentarier. Der neue Saarländische Landtag weist damit fünf Akteure auf, ist stärker als jemals zuvor fragmentiert und polarisiert. Seine neue Struktur erschwert die parlamentarische Arbeit und setzt die Regierungs- und Oppositionsparteien stärkerem Stress als früher aus.

Die Mandatsverteilung eröffnete für die Regierungsbildung theoretisch mehrere Möglichkeiten. Die Festlegungen im Wahlkampf, die ideologisch-programmatischen Distanzen sowie persönliche Antipathien schränkten die Optionen jedoch ein. Letzen Endes waren drei möglich: eine Große Koalition aus CDU und SPD, ein Bündnis aus SPD, Grünen und Linkspartei oder eine so genannte Jamaikakoalition aus CDU, FDP und Grünen. Für Peter Müller hieß die Alternative Große Koalition oder Jamaika, für Heiko Maas Große Koalition oder rot-rot-grün. Da weder Müller noch Maas eine Große Koalition anstrebten, blieben nur die beiden Varianten, bei denen den Grünen die Rolle als „Zünglein an der Waage“ zufiel. Zum zentralen, von allen umworbenen Entscheidungsträger wurde damit Hubert Ulrich.

Während die CDU schon im Wahlkampf etwas auf die Grünen zugegangen war, hatte die FDP auch aus Rücksicht auf die Bundespartei die Programme von Grünen und Freidemokraten für unvereinbar erklärt. Schon am Tag nach der Landtagswahl machte die FDP jedoch einen großen Schritt auf die Grünen zu. So sah ihr stellvertretender Landesvorsitzender Oliver Luksic nun große Schnittmengen, vor allem in der Wirtschafts- und Bildungspolitik sowie in Fragen der Bürgerrechte. Größere Zugeständnisse der CDU und der FDP an die Grünen und persönliche Antipathien zwischen den Akteuren der Grünen und der Linkspartei ebneten schließlich den Weg nach Jamaika.

Mit den Stimmen von CDU, FDP und Grünen wurde Peter Müller schließlich erwartungsgemäß zum ersten Regierungschef eines schwarz-gelb-grünen Bündnisses in Deutschland gewählt. An ihn sind hohe Erwartungen gerichtet, denn er muss vor allem zeigen, dass Jamaika auch in anderen Bundesländern als Regierungsoption möglich ist. Sollte die $\mathrm{Zu}-$ sammenarbeit von CDU und Grünen in Fragen des Umweltschutzes funktionieren, ist es sehr wahrscheinlich, dass noch bestehende Vorbehalte auf Seiten der Grünen bezüglich zukünftiger Beteiligungen an CDU-geführten Regierungen abgebaut werden. Stärker noch als früher wird Müller in der Union ein Gegengewicht zum konservativen hessischen CDULandesvorsitzenden und Ministerpräsidenten Roland Koch bilden.

Neben Peter Müller (CDU) als Ministerpräsident und Minister für Justiz gehören der neuen Landesregierung vier CDU-, zwei FDP- und zwei Minister der Grünen an (vgl. Tabelle 4), wobei Peter Jacoby, Karl Rauber und Annegret Kramp-Karrenbauer auch schon in der vorangegangenen Wahlperiode diese Ämter bekleideten.

Vorsitzender der CDU-Fraktion im Saarländischen Landtag ist Klaus Meiser; die FDPFraktion wird von Horst Hinschberger und die Fraktion von Bündnis90/Die Grünen von Hubert Ulrich geführt. Ihnen stehen auf Seiten der Opposition Heiko Maas als Vorsitzender 


\begin{tabular}{|l|l|}
\hline Tabelle 4: Das saarländische Kabinett nach der Wahl 2009 \\
\hline Ministerpräsident und Minister der Justiz & Peter Müller (CDU) \\
\hline $\begin{array}{l}\text { Stellvertretender Ministerpräsident und Minister } \\
\text { für Wirtschaft, Wissenschaft und Landwirtschaft }\end{array}$ & Christoph Hartmann (FDP) \\
\hline $\begin{array}{l}\text { Minister für Bundesangelegenheiten, Kultur } \\
\text { und Chef der Staatskanzlei }\end{array}$ & Karl Rauber (CDU) \\
\hline Minister der Finanzen & Peter Jacoby (CDU) \\
\hline Minister für Inneres und Europangelegenheiten & Stephan Toscani (CDU) \\
\hline $\begin{array}{l}\text { Ministerin für Arbeit, Familie, Soziales, Prävention } \\
\text { und Sport }\end{array}$ & Annegret Kramp-Karrenbauer (CDU) \\
\hline Minister für Gesundheit und Verbraucherschutz & Georg Weisweiler (FDP) \\
\hline Minister für Bildung & Klaus Kessler (Bündnis 90/Die Grünen) \\
\hline Ministerin für Umwelt, Energie und Verkehr & Simone Peter (Bündnis 90/Die Grünen) \\
\hline
\end{tabular}

der SPD-Fraktion und Oskar Lafontaine als Vorsitzender der Fraktion Die Linke gegenüber. Starke Antipathien zwischen beiden Akteuren dürften die Oppositionsarbeit auch deshalb erschweren, weil beide etwa gleich starke Fraktionen hinter sich haben und die Rolle als Oppositionsführer anstreben werden.

In seiner Regierungserklärung am 18. November 2009 schwor Müller die Saarländer auf schwere Zeiten ein. Erst jetzt gestand er ein, dass sich das Land in einer tiefen Krise befinde und deutete an, dass die wirtschaftliche Lage sich auch noch verschlechtern könne. Die Landesregierung werde jedoch alle Möglichkeiten ausschöpfen, die Arbeitslosigkeit zu begrenzen. Sie müsse sich vor allem drei Herausforderungen stellen: der Bewältigung der Wirtschaftsund Finanzkrise, der demographischen Entwicklung und dem Klimawandel. Während die ersten beiden Themen bereits in seiner Regierungserklärung nach der gewonnenen Landtagswahl 2004 hervorgehoben worden waren, ist das letzte Thema augenscheinlich ein Zugeständnis an die Regierungsbeteiligung der Grünen, die damit ein Kernthema ihres Wahlkampfes abdecken konnten ${ }^{28}$. Darüber hinaus teilte Müller den Saarländern mit, dass Bildung einen Schwerpunkt der Regierungsarbeit darstellen werde und die Hochschulen mehr Geld erhalten sollen. Jedem Kind und Jugendlichen sollten die gleichen Bildungs- und Aufstiegschancen geboten werden. Der Bildungsbereich werde deshalb von den Sparmaßnahmen ausgenommen, womit sich Forderungen der FDP und der Grünen durchsetzen konnten. Damit hat Peter Müller zugleich die Kriterien geliefert, an der die Amtszeit der neuen Regierung bei der nächsten Landtagswahl gemessen werden wird. Unabhängig davon, ob sie den gesellschaftlichen Herausforderungen gerecht werden kann und die Lebenschancen der Saarländer optimieren wird, stellt die Bildung der ersten Jamaikakoalition im politischen System der Bundesrepublik Deutschland eine Weichenstellung dar, die die überkommene politische Farbenlehre revidiert und das traditionelle Lagerdenken zu überbrücken hilft.

SPD und Linkspartei bilden die neue Opposition. Das Verhalten der Sozialdemokraten in der laufenden Wahlperiode wird maßgeblich davon abhängen, wie sie sich eine Regierungsbeteiligung nach der nächsten Landtagswahl vorstellen. Planen sie ein rot-rotes Bünd-

28 Vgl. „Neue Wege für ein modernes Saarland - Den Fortschritt nachhaltig gestalten”. Regierungserklärung des saarländischen Ministerpräsidenten Peter Müller vor dem Landtag des Saarlandes am 18. November 2009. 
nis, werden sie mit der Linkspartei eng zusammenarbeiten und eine inhaltliche und personelle Alternative im Saarland aufbauen müssen, ohne sich dabei zu weit der Linkspartei anzunähern. Voraussetzung dafür ist ein Rückzug von Lafontaine aus der Landespolitik. Hofft die SPD jedoch, die Grünen und eventuell auch die FDP ins Boot zu holen, wird sie nur moderate Kritik an der Regierungsarbeit der beiden Parteien üben können; andernfalls werden diese auf Distanz zu ihr gehen, was wiederum ihre Chancen bei den kommenden Wahlen schmälern dürfte.

\section{Zusammenfassung}

(1) Aufgrund der strukturellen Gegebenheiten und der politischen Traditionen befindet sich die CDU im Saarland seit Jahrzehnten in einer günstigeren Ausgangslage als die SPD. Wegen der anhaltenden Strukturkrise, aktueller Entwicklungen und Rahmenbedingungen startete die regierende CDU diesmal jedoch aus einer schlechten Ausgangsposition.

(2) Der Eintritt der vom früheren saarländischen Ministerpräsidenten Oskar Lafontaine geführten Linkspartei in den politischen Wettbewerb reduzierte die Chancen der oppositionellen SPD, an der Saar die Regierungsmacht zu übernehmen.

(3) Während die unter Druck stehende CDU einen Freund-Feind-Wahlkampf führte und die SPD mit der Abgrenzung zur Linkspartei zu kämpfen hatte, konnten FDP, Grüne und Linkspartei ihre teilweise diametral entgegengesetzten Positionen deutlich machen.

(4) Kennzeichnend für den Ausgang der Landtagswahl ist eine im Vergleich zu 2004 wieder angestiegene Wahlbeteiligung. Vor allem die Linkspartei konnte einen beträchtlichen Teil der früheren Nichtwähler mobilisieren.

(5) Im neuen Landtag sind fünf Parteien vertreten. Die CDU kommt auf nur noch 19 Mandate, bleibt aber stärkste Partei. Die SPD verliert abermals und stellt nur noch 13 Abgeordnete. Die FDP verfügt über fünf Sitze. Die Grünen erhalten erneut drei Mandate. Die Linkspartei zieht mit elf Abgeordneten erstmals in das Parlament ein.

(6) Im Saarland besteht nun ein asymmetrisches Fünfparteiensystem mit der CDU als immer noch dominanter politischer Kraft.

(7) Die CDU musste zwar gewaltige Stimmen- und Mandatsverluste verkraften, stellt aber erneut den Regierungschef. Ursache für den Niedergang der Christdemokraten sind eine gesunkene Reputation ihres Ministerpräsidenten, eine vergleichsweise schlechte Regierungsarbeit sowie eine verfehlte, auf Feindbilder setzende Wahlkampfstrategie.

(8) Obwohl die SPD und ihr Spitzenkandidat diesmal besser als 2004 bewertet wurden, wanderten erneut überdurchschnittlich viele Arbeiter, Arbeitslose und Gewerkschaftsmitglieder von ihr ab. Die Bedingungen für einen Wiederaufstieg der SPD an der Saar sind ungünstiger als je zuvor.

(9) Die Linkspartei ist der große Gewinner der Wahl. Sie profitierte von einem herausragenden Spitzenkandidaten und den Nachwirkungen der Regierungspolitik der SPD im Bund. Mit ihren sozialpolitischen Forderungen konnte sie traditionelle Wählergruppen der SPD mobilisieren. Ihr Sieg gibt der Linkspartei in anderen westlichen Bundesländern Auftrieb.

(10)Bündnis 90/Die Grünen sind die Königsmacher an der Saar. Ihnen verdankt Peter Müller sein Verbleiben im Amt des Ministerpräsidenten. Die erste Jamaikakoalition stellt eine Zäsur in der politischen Farbenlehre der Bundesrepublik Deutschland dar. 